\title{
Replication of genetic variants from genome-wide association studies with metabolic traits in an island population of the Adriatic coast of Croatia
}

\author{
Rebekah Karns ${ }^{1}$, Ge Zhang ${ }^{1,2}$, Nina Jeran ${ }^{3}$, Dubravka Havas-Augustin ${ }^{3}$, Sasa Missoni ${ }^{3}$, Wen Niu ${ }^{1}$, \\ Subba Rao Indugula ${ }^{1}$, Guangyun Sun ${ }^{1}$, Zijad Durakovic ${ }^{3}$, Nina Smolej Narancic ${ }^{3}$, Pavao Rudan ${ }^{3}$, \\ Ranajit Chakraborty ${ }^{4}$ and Ranjan Deka ${ }^{\star, 1}$
}

\begin{abstract}
Twenty-two single-nucleotide polymorphisms (SNPs) in 10 gene regions previously identified in obesity and type 2 diabetes (T2D) genome-wide association studies (GWAS) were evaluated for association with metabolic traits in a sample from an island population of European descent. We performed a population-based study using 18 anthropometric and biochemical traits considered as continuous variables in a sample of 843 unrelated subjects ( 360 men and 483 women) aged 18-80 years old from the island of Hvar on the eastern Adriatic coast of Croatia. All eight GWAS SNPs in FTO were significantly associated with weight, body mass index, waist circumference and hip circumference; 20 of the 32 nominal $P$-values remained significant after permutation testing for multiple corrections. The strongest associations were found between the two TCF7L2 GWAS SNPs with fasting plasma glucose and $\mathrm{HbAlc}$ levels, all four $P$-values remained significant after permutation tests. Nominally significant associations were found between several SNPs and other metabolic traits; however, the significance did not hold after permutation tests. Although the sample size was modest, our study strongly replicated the association of $F T O$ variants with obesity-related measures and TCF7L2 variants with T2D-related traits. The estimated effect sizes of these variants were larger or comparable to published studies. This is likely attributable to the homogenous genetic background of the relatively isolated study population.
\end{abstract}

European Journal of Human Genetics (2011) 19, 341-346; doi:10.1038/ejhg.2010.178; published online 8 December 2010

Keywords: genetic association; obesity; type 2 diabetes; FTO; TCF7L2; isolated population

\section{INTRODUCTION}

Recent genome-wide association studies (GWAS) have identified nearly 30 common sequence variants associated with obesity and type 2 diabetes (T2D) related traits in populations primarily of European descent. Genes that have been implicated in T2D harboring these variants include TCF7L2, SLC3OA8, HHEX/IDE, CDKAL1, $C D K N 2 A / B, I G F 2 B P 2, T C F 2$ and PPARG; ${ }^{1-7}$ genes that are primarily associated with obesity-related phenotypes are INSIG2, FTO, MC4R, $B D N F$ and SH2B1. ${ }^{8-14}$ Among these genes, TCF7L2 and FTO have emerged as the strongest candidates conferring risks to T2D and obesity, respectively, replicated across studies with relatively large effect sizes. ${ }^{15,16}$ The purpose of this study was to replicate a set of the significant GWAS single-nucleotide polymorphisms (SNPs) from the aforementioned studies with several metabolic traits in an island population from the eastern Adriatic coast of Croatia.

Croatian islanders are primarily of Slavic descent, who emigrated from the mainland at successive time periods, the latest during fifteenth and eighteenth century AD following the Turkish invasions. ${ }^{17,18}$ Since that time, the populations have remained relatively isolated largely because of their geographic isolation with minimal immigration from the mainland. In spite of sharing a common descent, these populations are distinct from their mainland European ancestors, practicing a traditional life-style pattern based primarily on an agricultural subsistence in a rural setting and living on a typical 'Mediterranean' diet of fish, vegetables, olive oil and red wine. ${ }^{18}$ However, previous studies have shown high prevalence of obesity and hypertension, ${ }^{19-21}$ which imply factors other than nutritional practices and life-style patterns have influenced variations in metabolic profiles of these populations.

We analyzed 22 significant GWAS SNPs located in and around INSIG2, PPARG, CDKAL1, SLC30A8, CDKN2A, CDKN2B, HHEX/ IDE, TCF7L2, FTO and MC4R to test for associations with traits that include obesity-related measures of body mass index (BMI), waist circumference (WC), hip circumference (HC), waist-hip ratio (WHR), blood pressure and biochemical measures of fasting plasma glucose (FPG), HbAlc, insulin, HDL, LDL, total cholesterol (TC), triglyceride (TG), fibrinogen, calcium, creatinine and uric acid levels. Among the significant observations, we found strong associations of two SNPs (rs7903146 and rs12255372) in TCF7L2 with FPG and HbAlc levels; and all eight SNPs in FTO were individually associated with weight, BMI, WC and $\mathrm{HC}$.

${ }^{1}$ Department of Environmental Health, University of Cincinnati College of Medicine, Cincinnati, OH, USA; ${ }^{2}$ Department of Family and Community Medicine, University of Cincinnati College of Medicine, Cincinnati, OH, USA; ${ }^{3}$ Institute for Anthropological Research, Zagreb, Croatia; ${ }^{4}$ Center for Computational Genomics, Institute of Investigative Genetics, University of North Texas Health Science Center, Fort Worth, TX, USA

${ }^{*}$ Correspondence: Professor R Deka, Department of Environmental Health, University of Cincinnati College of Medicine, Cincinnati, OH 45267-0056, USA.

Tel: +1 513558 5989; Fax: +1 513558 4397; E-mail: ranjan.deka@uc.edu

Received 8 March 2010; revised 27 September 2010; accepted 7 October 2010; published online 8 December 2010 


\section{MATERIALS AND METHODS}

\section{Study population and phenotypic measurements}

The study samples were collected from the island of Hvar, the largest of the middle Dalmatian islands on the Adriatic coast with a population of over 11500 individuals. Anthropometric measurements of height (Ht), weight (Wt), WC, HC, blood pressure and blood samples were obtained from 843 unrelated individuals (360 men and 483 women) aged 18 to 80 years old from the island of Hvar. Subjects within this age bracket were randomly ascertained without consideration for any known disease status and use of medication. Study protocols were approved by the ethics committee of the Institute for Anthropological Research, Zagreb and the Institutional Review Board of the University of Cincinnati.

Data on $\mathrm{Ht}, \mathrm{Wt}, \mathrm{WC}$ and $\mathrm{HC}$ were used to compute the composite scores of BMI (Wt in $\mathrm{kg}) /\left(\mathrm{Ht}\right.$ in $\left.\mathrm{m}^{2}\right)$, and WHR (WC/HC). Blood pressure was measured three times using mercury sphygmomanometer at resting position; the second and the third measurements were used to calculate the mean systolic (SBP) and diastolic (DBP) blood pressure. Blood samples were drawn after a 12-h fast and serum was separated and kept frozen until shipped to the clinical chemistry laboratory in Zagreb, where biochemical tests were performed to measure FPG, HbAlc, insulin, HDL, LDL, TC, TG, fibrinogen, calcium, creatinine and uric acid levels using standard methods.

\section{Genotyping}

Twenty-two SNPs (Table 1) significantly associated with anthropometric and biochemical traits in GWAS were genotyped in the total sample of 843 subjects. The five MC4R SNPs were genotyped using the TaqMan assay and the remaining 17 SNPs were typed using the SNPlex assay following protocols described previously.22 Quality control was assured by introducing blind duplicates and negative controls into each batch of samples in the 96-well format. The overall genotype call rate of the 22 SNPs was $96.8 \%$ and the genotypic consistency rate based on 8 internal replicates was $>99.5 \%$.

\section{Statistical analysis}

Descriptive statistics of the phenotypic traits were calculated using SAS v.9. 1 (SAS Institute, Inc., Cary, NC, USA). Allele and genotype frequencies were computed using the HelixTree software (Golden Helix, Inc., Bozeman, MT, USA). Hardy-Weinberg equilibrium (HWE) was assessed by Fisher's exact test. The phenotypic traits were adjusted for the effects of age, gender and their interaction term by linear regression. The association between each SNP and the adjusted traits was performed using HelixTree based on an additive model ( $1 \mathrm{df}$ test) with $P$-values considered nominally significant at $\leq 0.05$. A permutation-based analysis, with 100000 iterations, was performed to account for multiple tests based on the number of markers.

\section{RESULTS}

Table 1 shows the description of the examined SNPs with their NCBI dbSNP IDs ('rs' numbers), gene location, chromosomal base positions and minor allele frequencies (MAFs). Genotype proportions at all SNPs conformed to the expectations of HWE with a $P$-value $>0.01$ (data not shown). All of the SNPs in our population are moderately to highly polymorphic with the MAF ranging between 0.13 and 0.49 . In general, the allele frequencies corresponded to those in the European HapMap sample (www.HapMap.org)

Descriptive statistics (mean $\pm \mathrm{SD}$ ) of the phenotypic traits are presented in Table 2. The mean age of the participants at the time of sampling was 57.84 years (59.55 years in men and 56.56 years in women). All of the examined phenotypes were considered as continuous traits for analysis. Of the two classic measures of obesity, mean BMI was within the normal range of variation $\left(\mathrm{BMI} \leq 30 \mathrm{~kg} / \mathrm{m}^{2}\right)$ in both genders; however, mean WC was above the normal range as defined either by the ATPIII (Adult Treatment Panel III) ${ }^{23}$ or IDF

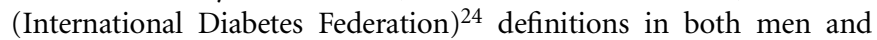

Table 1 Summary of the studied GWAS SNPs

\begin{tabular}{|c|c|c|c|c|c|c|c|c|c|}
\hline$N C B I S N P I D$ & Gene & Chromosome & Position $^{\mathrm{a}}$ & $\begin{array}{l}\text { Study } \\
\text { reference }\end{array}$ & $\begin{array}{l}\text { Ancestry of reference } \\
\text { population }\end{array}$ & $\begin{array}{c}\text { Phenotype } \\
\text { of reference } \\
\text { study }\end{array}$ & Alleles ${ }^{\mathrm{b}}$ & $\begin{array}{c}\text { HapMap } \\
\text { caucasian } \\
\text { MAF }\end{array}$ & $M A F^{\mathrm{C}}$ \\
\hline rs7566605 & INSIG2 & $2 q 14$ & $1.19 \mathrm{E}+08$ & Herbert et $a l^{8}$ & $\begin{array}{l}\text { Western European } \\
\text { African American }\end{array}$ & Obesity & CG & 0.26 & 0.4 \\
\hline rs1801282 & PPARG & $3 p 25$ & 12368125 & WTCCC ${ }^{7}$ & Western European & $\mathrm{T} 2 \mathrm{D}$ & CG & 0.10 & 0.16 \\
\hline rs10946398 & CDKAL1 & $6 p 22$ & 20769013 & Zeggini et $a A^{A}$ & Western European & $\mathrm{T} 2 \mathrm{D}$ & $A C$ & 0.34 & 0.3 \\
\hline rs13266634 & SLC30A8 & $8 q 24$ & $1.18 \mathrm{E}+08$ & Sladek et $a l^{1}$ & Western European & $\mathrm{T} 2 \mathrm{D}$ & CT & 0.24 & 0.36 \\
\hline rs564398 & $C D K N 2 B$ & $9 p 21$ & 22019547 & Zeggini et $a A^{4}$ & Western European & $\mathrm{T} 2 \mathrm{D}$ & $A G$ & 0.43 & 0.39 \\
\hline rs10811661 & $C D K N 2 A$ & $9 p 21$ & 22124094 & $\left.D G\right|^{3}$ & Western European & $\mathrm{T} 2 \mathrm{D}$ & TC & 0.20 & 0.13 \\
\hline rs1111875 & HHEX-IDE & $10 q 23$ & 94452862 & Sladek et $a l^{1}$ & Western European & $\mathrm{T} 2 \mathrm{D}$ & $\mathrm{GA}$ & 0.42 & 0.42 \\
\hline rs7903146 & TCF7L2 & $10 q 25$ & $1.15 \mathrm{E}+08$ & Grant et aR9 & Western European & Obesity & TC & 0.28 & 0.29 \\
\hline rs12255372 & TCF7L2 & $10 q 25$ & $1.15 \mathrm{E}+08$ & Grant et aR9 & Western European & Obesity & GT & 0.25 & 0.28 \\
\hline rs9939973 & FTO & $16 q 12$ & 52358069 & Hinney et al ${ }^{11}$ & Western European & Obesity & $\mathrm{GA}$ & 0.48 & 0.49 \\
\hline rs1421085 & FTO & $16 q 12$ & 52358455 & Dina et $a l^{10}$ & Western European & Obesity & TC & 0.46 & 0.47 \\
\hline rs1121980 & FTO & $16 q 12$ & 52366748 & Dina et $a l^{10}$ & Western European & Obesity & CT & 0.48 & 0.47 \\
\hline rs17817449 & FTO & $16 q 12$ & 52370868 & Dina et $a l^{10}$ & Western European & Obesity & $\mathrm{TG}$ & 0.46 & 0.44 \\
\hline rs8050136 & FTO & $16 q 12$ & 52373776 & Grant et aR6 & Western European & Obesity & $\mathrm{CA}$ & 0.46 & 0.44 \\
\hline rs3751812 & FTO & $16 q 12$ & 52375961 & Dina et $a l^{10}$ & Western European & Obesity & GT & 0.46 & 0.44 \\
\hline rs9939609 & FTO & $16 q 12$ & 52378028 & Frayling et $a \rho$ & Western European & Obesity & $\mathrm{TA}$ & 0.46 & 0.43 \\
\hline rs7190492 & FTO & $16 q 12$ & 52386253 & Grant et aR6 & Western European & Obesity & GA & 0.35 & 0.33 \\
\hline rs17782313 & $M C 4 R$ & $18 q 22$ & 56002077 & Loos et all12 & Western European & Obesity & TC & 0.26 & 0.23 \\
\hline rs12970134 & $M C 4 R$ & $18 q 22$ & 56035730 & Chambers et $a l^{13}$ & Indian Asian, European & T2D-related traits ${ }^{d}$ & GA & 0.28 & 0.22 \\
\hline rs477181 & $M C 4 R$ & $18 q 22$ & 56047018 & Chambers et al ${ }^{13}$ & Indian Asian, European & T2D-related traits ${ }^{\mathrm{d}}$ & GT & 0.34 & 0.33 \\
\hline rs502933 & $M C 4 R$ & $18 q 22$ & 56047454 & Chambers et al ${ }^{13}$ & Indian Asian, European & T2D-related traits ${ }^{\mathrm{d}}$ & $\mathrm{CA}$ & 0.34 & 0.34 \\
\hline rs4450508 & $M C 4 R$ & $18 q 22$ & 56064414 & Chambers et al ${ }^{13}$ & Indian Asian, European & T2D-related traits ${ }^{\mathrm{d}}$ & $\mathrm{GA}$ & 0.38 & 0.35 \\
\hline
\end{tabular}

${ }^{a}$ Chromosomal base position with reference to Human Genome Build 36.3.

becond nucleotide corresponds to the minor allele.

cMinor allele frequency (MAF) in this study population.

dHOMA-IR, WC, WHR, Wt, BMI, HDL, TG, DBP, T2D and metabolic syndrome. 
Table 2 Summary statistics of the phenotypic traits

\begin{tabular}{|c|c|c|c|c|c|c|c|c|c|c|c|c|}
\hline \multirow[b]{2}{*}{ Trait } & \multicolumn{4}{|c|}{ Male } & \multicolumn{4}{|c|}{ Female } & \multicolumn{4}{|c|}{ Total sample } \\
\hline & $\mathrm{N}$ & $M e a n \pm S D$ & Min & Max & $\mathrm{N}$ & $M e a n \pm S D$ & Min & Max & $\mathrm{N}$ & $M e a n \pm S D$ & Min & $\operatorname{Max}$ \\
\hline Age (year) & 360 & $59.55 \pm 12.46$ & 19.9 & 80 & 483 & $56.56 \pm 13.23$ & 18.1 & 79.8 & 843 & $57.84 \pm 12.98$ & 18.1 & 80 \\
\hline $\mathrm{Ht}(\mathrm{cm})$ & 360 & $177.42 \pm 7.22$ & 157.2 & 206 & 483 & $164.10 \pm 6.78$ & 139 & 187.2 & 843 & $169.78 \pm 9.59$ & 139 & 206 \\
\hline Wt (kg) & 360 & $89.31 \pm 12.93$ & 60.2 & 142.5 & 483 & $74.10 \pm 12.69$ & 43 & 123.9 & 843 & $80.59 \pm 14.84$ & 43 & 142.5 \\
\hline BMI $\left(\mathrm{kg} / \mathrm{m}^{2}\right)$ & 360 & $28.32 \pm 3.34$ & 19.64 & 42.23 & 483 & $27.54 \pm 4.58$ & 16.7 & 45.79 & 843 & $27.87 \pm 4.11$ & 16.7 & 45.79 \\
\hline WC $(\mathrm{cm})$ & 359 & $101.85 \pm 8.98$ & 79.3 & 138.5 & 482 & $91.90 \pm 12.09$ & 62.3 & 137.4 & 841 & $96.15 \pm 11.93$ & 62.3 & 138.5 \\
\hline $\mathrm{HC}(\mathrm{cm})$ & 360 & $104.42 \pm 7.29$ & 90.8 & 132 & 482 & $106.15 \pm 10.46$ & 75 & 150 & 842 & $105.41 \pm 9.27$ & 75 & 150 \\
\hline WHR & 359 & $0.97 \pm 0.05$ & 0.727 & 1.124 & 482 & $0.86 \pm 0.06$ & 0.651 & 1.12 & 841 & $0.91 \pm 0.08$ & 0.651 & 1.124 \\
\hline DBP (mm Hg) & 360 & $84.91 \pm 9.20$ & 60 & 120 & 483 & $81.49 \pm 10.47$ & 55 & 115 & 843 & $82.95 \pm 10.08$ & 55 & 120 \\
\hline $\mathrm{SBP}(\mathrm{mm} \mathrm{Hg})$ & 360 & $138.59 \pm 20.56$ & 100 & 240 & 483 & $132.42 \pm 24.46$ & 90 & 230 & 843 & $135.05 \pm 23.07$ & 90 & 240 \\
\hline $\mathrm{FPG}(\mathrm{mmol} / \mathrm{l})$ & 360 & $6.26 \pm 1.40$ & 4.2 & 16.8 & 483 & $5.86 \pm 1.37$ & 4.1 & 14.9 & 843 & $6.03 \pm 1.39$ & 4.1 & 16.8 \\
\hline HbAlc (\%) & 360 & $5.78 \pm 0.84$ & 4.6 & 12 & 482 & $5.77 \pm 0.87$ & 4.7 & 12.4 & 842 & $5.77 \pm 0.86$ & 4.6 & 12.4 \\
\hline Insulin (mmol/l) & 360 & $11.15 \pm 8.13$ & 1.15 & 72.26 & 482 & $11.383 \pm 7.93$ & 1.3 & 56.26 & 842 & $11.28 \pm 8.01$ & 1.15 & 72.26 \\
\hline $\mathrm{HDL}(\mathrm{mmol} / \mathrm{l})$ & 360 & $1.30 \pm 0.34$ & 0.2 & 3.55 & 482 & $1.53 \pm 0.39$ & 0.77 & 4.61 & 842 & $1.43 \pm 0.38$ & 0.2 & 4.61 \\
\hline LDL (mmol/l) & 358 & $3.68 \pm 0.95$ & 1.1 & 6.7 & 481 & $3.91 \pm 1.04$ & 1.2 & 8 & 839 & $3.81 \pm 1.01$ & 1.1 & 8 \\
\hline $\mathrm{TC}(\mathrm{mmol} / \mathrm{l})$ & 360 & $5.80 \pm 1.10$ & 2.4 & 9.1 & 482 & $6.09 \pm 1.22$ & 1.5 & 10.4 & 842 & $5.971 \pm 1.18$ & 1.5 & 10.4 \\
\hline $\mathrm{TG}(\mathrm{mmol} / \mathrm{l})$ & 360 & $1.70 \pm 1.03$ & 0.36 & 6.31 & 482 & $1.41 \pm 0.75$ & 0.33 & 5.09 & 842 & $1.53 \pm 0.89$ & 0.33 & 6.31 \\
\hline Fibrinogen $(\mathrm{mmol} / \mathrm{l})$ & 355 & $3.63 \pm 1.25$ & 1.3 & 8.1 & 478 & $4.03 \pm 1.38$ & 1.4 & 9.2 & 833 & $3.86 \pm 1.34$ & 1.3 & 9.2 \\
\hline Calcium (mmol/l) & 358 & $2.36 \pm 0.13$ & 1.66 & 3.2 & 482 & $2.37 \pm 0.11$ & 1.75 & 2.9 & 840 & $2.36 \pm 0.12$ & 1.66 & 3.2 \\
\hline Creatinine $(\mathrm{mmol} / \mathrm{l})$ & 360 & $97.68 \pm 20.94$ & 54 & 280 & 482 & $78.84 \pm 12.99$ & 48 & 141 & 842 & $86.89 \pm 19.26$ & 48 & 280 \\
\hline Uric Acid (mmol/l) & 359 & $365.89 \pm 80.58$ & 169 & 671 & 482 & $265.32 \pm 73.09$ & 77 & 632 & 841 & $308.25 \pm 91.12$ & 77 & 671 \\
\hline
\end{tabular}

Table 3a $P$-values of single-locus association tests with traits of primary involvement with obesity and T2D

\begin{tabular}{|c|c|c|c|c|c|c|c|c|c|}
\hline$S N P$ & Gene & $W t$ & $B M I$ & $W C$ & $H C$ & $W H R$ & $F P G$ & $H b A 1 c$ & Insulin \\
\hline rs7566605 & INSIG2 & 0.8241 & 0.6250 & 0.6983 & 0.8846 & 0.4685 & 0.7412 & 0.3214 & 0.3683 \\
\hline rs1801282 & PPARG & 0.9015 & 0.9478 & 0.7123 & 0.2166 & 0.4526 & 0.0463 & 0.0402 & 0.8157 \\
\hline rs10946398 & CDKAL1 & 0.4687 & 0.7888 & 0.8522 & 0.8098 & 0.6234 & 0.5921 & 0.0380 & 0.0809 \\
\hline rs13266634 & SLC30A8 & 0.3859 & 0.8006 & 0.5610 & 0.9760 & 0.3627 & 0.7612 & 0.3965 & 0.3110 \\
\hline rs564398 & $C D K N 2 B$ & 0.2659 & 0.5976 & 0.3366 & 0.3005 & 0.6022 & 0.7524 & 0.8627 & 0.2888 \\
\hline rs10811661 & $C D K N 2 A$ & 0.7389 & 0.6424 & 0.3315 & 0.8007 & 0.3216 & 0.0426 & 0.0336 & 0.6773 \\
\hline rs1111875 & HHEX/IDE & 0.0289 & 0.0413 & 0.1624 & 0.2168 & 0.5880 & 0.0164 & 0.0163 & 0.4383 \\
\hline rs7903146 & TCF7L2 & 0.1785 & 0.2602 & 0.3611 & 0.5749 & 0.4348 & $1.29 \mathrm{E}-07$ & $1.14 \mathrm{E}-09$ & 0.4202 \\
\hline rs12255372 & TCF7L2 & 0.1930 & 0.0950 & 0.2746 & 0.3026 & 0.5428 & $\overline{9.62 \mathrm{E}-08}$ & $\overline{6.37 \mathrm{E}-09}$ & 0.1310 \\
\hline rs9939973 & FTO & 0.0024 & 0.0047 & 0.0009 & 0.0139 & 0.0305 & 0.9428 & 0.3743 & 0.0167 \\
\hline rs1421085 & FTO & 0.0013 & 0.0010 & 0.0004 & 0.0010 & 0.1277 & 0.9686 & 0.4779 & 0.0664 \\
\hline rs1121980 & FTO & $\overline{0.0010}$ & $\overline{0.0013}$ & $\overline{0.0006}$ & $\overline{0.0022}$ & 0.1169 & 0.8934 & 0.5187 & 0.0457 \\
\hline rs17817449 & FTO & $\overline{0.0009}$ & 0.0025 & 0.0009 & $\overline{0.0021}$ & 0.1462 & 0.5949 & 0.8865 & 0.1571 \\
\hline rs8050136 & FTO & $\overline{0.0051}$ & $\overline{0.0179}$ & $\overline{0.0026}$ & $\overline{0.0078}$ & 0.1524 & 0.6895 & 0.7871 & 0.1714 \\
\hline rs3751812 & FTO & 0.0029 & 0.0064 & $\overline{0.0020}$ & 0.0029 & 0.2310 & 0.5585 & 0.9416 & 0.1086 \\
\hline rs9939609 & FTO & $\overline{0.0018}$ & 0.0043 & $\overline{0.0016}$ & $\overline{0.0063}$ & 0.1027 & 0.5013 & 0.7326 & 0.2418 \\
\hline rs7190492 & FTO & 0.0054 & 0.0130 & $\overline{0.0009}$ & 0.0110 & 0.0229 & 0.2070 & 0.4397 & 0.0768 \\
\hline rs17782313 & $M C 4 R$ & 0.0817 & 0.3312 & $\overline{0.1987}$ & 0.2315 & 0.5412 & 0.8683 & 0.1626 & 0.5135 \\
\hline rs12970134 & $M C 4 R$ & 0.0540 & 0.1401 & 0.1414 & 0.1822 & 0.4766 & 0.8727 & 0.4638 & 0.9882 \\
\hline rs477181 & $M C 4 R$ & 0.2032 & 0.4211 & 0.6506 & 0.1405 & 0.3210 & 0.9218 & 0.4512 & 0.5074 \\
\hline rs502933 & $M C 4 R$ & 0.0961 & 0.2539 & 0.3708 & 0.0711 & 0.4804 & 0.9472 & 0.4479 & 0.9060 \\
\hline rs4450508 & $M C 4 R$ & 0.1356 & 0.2424 & 0.3561 & 0.0918 & 0.5768 & 0.8037 & 0.6887 & 0.9428 \\
\hline
\end{tabular}

Empirical $P$-values $(<0.05)$ are shown in bold; $P$-values that maintain significance after permutation tests are underlined.

women $(\mathrm{men}=101.9 \mathrm{~cm}$, women $=91.9 \mathrm{~cm})$. Among the biochemical traits, mean FPG in men $(6.3 \mathrm{mmol} / \mathrm{l})$, TC in men $(5.8 \mathrm{mmol} / \mathrm{l})$ and women $(6.1 \mathrm{mmol} / \mathrm{l})$, and fibrinogen $(3.6 \mathrm{~g} / \mathrm{l}$ in men and $4.0 \mathrm{~g} / \mathrm{l}$ in women) were slightly above the normal cutoff values. With an expected correlation of these measures with age, a likely explanation for this observation is the study group represents a relatively older population as indicated by the mean age of the participants.
The results of genetic association of the 22 SNPs with the 18 traits are presented in Tables $3 \mathrm{a}$ and $\mathrm{b}$. In Table $3 \mathrm{a}$, we show the $P$-values of single-locus association tests with traits that are primarily associated with measures of obesity (Wt, BMI, WC, HC and WHR) and T2D (FPG, HbAlc and insulin). The most notable observations were with the SNPs in FTO and TCF7L2. All 8 FTO SNPs were individually associated with Wt, BMI, WC and HC; 20 of the 32 nominal $P$-values 
Table 3b $P$-values of single-locus association test with metabolic traits of secondary involvement with obesity and T2D

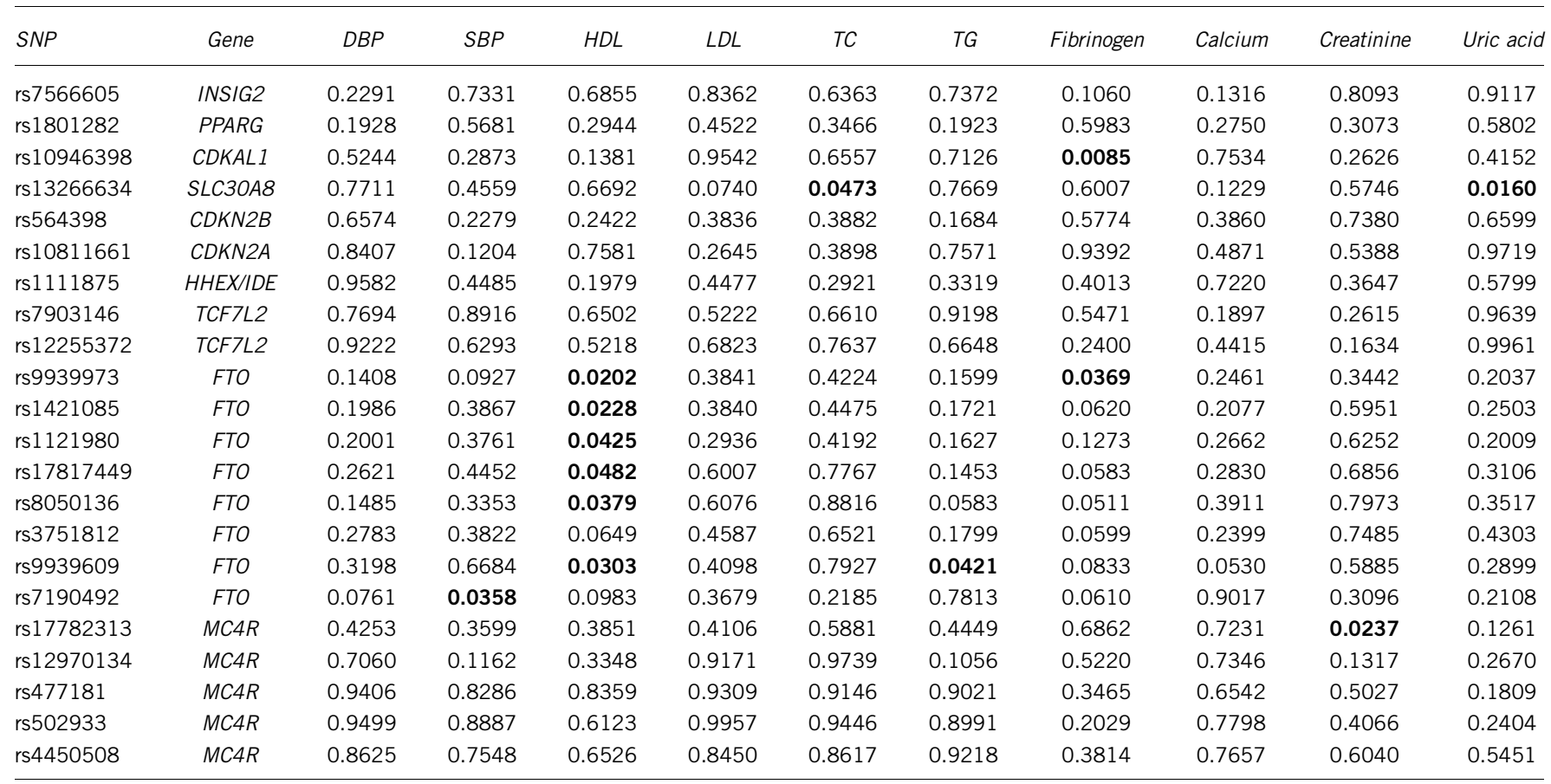

Empirical $P$-values $(<0.05)$ are shown in bold.

remained significant after permutation testing. Two FTO SNPs (rs9939973 and rs1121980) were also nominally associated with insulin. The strongest associations were found between the two TCF7L2 SNPs (rs7903146 and rs12255372) and FPG and HbAlc, with $P$-values (all $\leq 1 \times 10^{-5}$ ) remaining significant after the permutation tests. In addition, rs1801282 (PPARG), rs10811661 $(C D K N 2 A / B)$ and rs1111875 (HHEX/IDE) were marginally associated with both FPG and HbAlc levels. The HHEX/IDE variant was also nominally associated with Wt and BMI.

Table $3 \mathrm{~b}$ shows the results of single SNP associations with traits that are involved in the larger milieu of metabolic abnormalities and secondarily associated with obesity and T2D. Although the $P$-values are nominal, six FTO SNPs (rs9939973, rs1421085, rs1121980, rs17817449, rs8050136 and rs9939609) were associated with HDL; rs7190492 in FTO was associated with SBP; rs132266634 in SLC30A8 was associated with TC; rs9939609 in FTO was associated with TG; rs10946398 in CDKAL1 and rs9939973 in FTO were associated with fibrinogen; rs17782313 in MC4R was associated with creatinine; and rs13266634 in SLC30A8 was associated with uric acid.

\section{DISCUSSION}

We sought to replicate associations of 22 GWAS variants in 10 gene regions with a series of metabolic traits in an isolated population of European descent. The published studies have provided both direct and indirect explanations of biological plausibility of these genes/ regions (INSIG2, PPARG, HHEX/IDE, CDKAL1, SLC30A8, CDKN2A, CDKN2B, TCF7L2, FTO and MC4R) in the pathophysiology of carbohydrate intolerance and the maintenance of energy balance. Our study replicated the associations of FTO and TCF7L2 GWAS SNPs with obesity and T2D-related traits, respectively. The FTO gene variants have shown consistent associations with obesity across several studies. $^{9-11,14,25-27}$ These studies primarily considered BMI as the measure of obesity. We extended the list of the phenotypic traits influencing obesity and found without exception, FTO variants were associated with each of the four obesity-related traits (Wt, BMI, WC and HC, with $P$-values ranging from 0.018 to 0.0004 ). We found clear replication of previously reported associations of two SNPs, rs3751821 and rs1421085, with Wt and BMI, respectively. ${ }^{14,28}$ Overall, the consistent associations between the extended measures of obesity and all eight SNPs provide further evidence that FTO variants influence body fatness. In a recent study, we evaluated association of 29 SNPs on FTO, including the eight variants reported here, with a larger number of obesity-related phenotypic traits. ${ }^{29}$ In addition to the body fatness measures (BMI, Wt, WC and HC), this study showed that FTO variants influence lean mass (eg, bicondilar upper arm width), suggesting pleiotropic effects of the FTO gene. Also, we found that the body fatness measures were highly correlated (all $\left.r^{2}>0.8\right),{ }^{29}$ which at least partially explain the consistent association of the obesity-related traits with FTO SNPs. However, precise relationships between FTO SNPs and each of the obesity-related traits are difficult to assert from these observations, as the genetic and environmental contributions to their correlations are unknown.

With respect to the risk of $\mathrm{T} 2 \mathrm{D}$, the strongest evidence for association has been found with variants on the TCF7L2 gene with odds ratios $>1.5$ in multiple studies. ${ }^{1,2,30-32}$ We genotyped two GWAS SNPs, each showing very strong associations with FPG ( rs7903146, $P=1.29 \times 10^{-7} ;$ rs12255372, $P=9.62 \times 10^{-8}$ ) and HbAlc (rs7903146, $P=1.14 \times 10^{-9} ; \quad$ rs12255372, $P=6.37 \times 10^{-9}$ ). We also performed a secondary analysis of all SNPs taking into consideration FPG and HbAlc as dichotomous traits being surrogates for T2D state (all data not shown). In the total sample of 843 individuals, $113 \mathrm{had}$ FPG $\geq 126 \mathrm{mg}$ per $100 \mathrm{ml}$ and 100 subjects had HbAlc $\geq 6.5 \%$, which are cut points for T2D according to the World Health Organization, which are also used as standards for the Croatian populations. We found highly significant associations of the TCF7L2 SNPs with T2D status based on both FPG (rs7903146, $P=5.8 \times 10^{-5}$; rs12255372, $P=0.0001)$ and HbAlc (rs7903146, $P=9.93 \times 10^{-6}$; rs12255372, 
$P=3.33 \times 10^{-5}$ ), all $P$-values remained significant after corrections for multiple tests. In spite of the relatively smaller sample size of our study, these results reiterate the importance of genetic variation in FTO and TCF7L2 influencing the risk of obesity and T2D in our study population.

We found effect size estimates of variants in FTO on measures of body fatness were comparable to those reported in previous GWAS, which reported various FTO SNP effect sizes between 0.33 and $1.45 \mathrm{~kg} / \mathrm{m}^{2}$ per risk allele. ${ }^{9,14,25,28}$ For example, each copy of the C allele of the most significant FTO SNP rs1421085 was associated with an elevation of age and gender adjusted values of $\mathrm{Wt}(1.98 \mathrm{~kg}), \mathrm{HC}$ $(1.42 \mathrm{~cm})$, WC $(1.78 \mathrm{~cm})$ and BMI $\left(0.64 \mathrm{~kg} / \mathrm{m}^{2}\right)$. Under the additive model, the variance explained by rs1421085 was $1.22 \%(\mathrm{Wt}), 1.27 \%$ (HC), $1.49 \%$ (WC) and 1.29\% (BMI). An example of this is presented graphically in Supplementary Figure 1 illustrating the comparability of effect sizes (in BMI units) between this study and previously reported GWAS based on rs1421085 and SNPs that are in strong linkage disequilibrium (LD) with this SNP in our population. The effect sizes obtained by Dina et al ${ }^{10}$ are somewhat larger, which is likely because of their use of morbidly obese individuals in effect size calculations. Importantly, the most significant SNP in our study, rs1421085, overlaps the $95 \%$ confidence intervals of SNPs in high LD in the reported GWAS. Similarly, in TCF7L2, each copy of the $\mathrm{T}$ allele of rs12255372 corresponded with an elevation of age and gender adjusted values of FPG $(0.39 \mathrm{mmol} / \mathrm{l})$ and $\mathrm{HbAlc}(0.27 \%)$. The variance explained by the effect of rs12255372 was $4.4 \%$ (FPG) and $4.3 \%$ (HbAlc). As noted above, in GWAS FTO and TCF7L2 have emerged as the most important genes influencing the risks for obesity and $\mathrm{T} 2 \mathrm{D}$ and have been replicated in populations of diverse origins. Our results of association not only confirmed these findings, but were further augmented by higher effect sizes of the variants, which is likely attributable to a homogenous genetic background of the study population. It should also be noted that the eight FTO SNPs in our study population were in high LD, with average pairwise $r^{2}>0.8$ and the two TCF7L2 SNPs were in near perfect LD $\left(r^{2}=0.92\right)$.

The modest sample size of our study limited the power to detect signals of association of variants of small effect sizes or to provide confirmation of previously reported findings. On the basis of power calculations for 22 SNPs, however, we have sufficient power to detect SNPs with moderate effect size. For example, our study has $94.8 \%$ power to detect SNPs, which explain $1.5 \%$ variance at the nominal significance level $(\alpha=0.05)$. Even using the multiple-testing adjusted significance level $(\alpha=0.05 / 14,14$ is the estimated number of equivalent independent tests of the 22 SNPs based on permutation), the power is $74.8 \%$. We found nominal replications of the SNPs on PPARG (rs1801282), CDKN2A/B (rs10811661) and HHEX/IDE (rs111875) with both FPG and HbAlc levels. Each of these three SNPs was previously associated with T2D risk in GWAS; ${ }^{4,7}$ direction of associations in our study was consistent with these studies. Of note is the PPARG SNP rs1801282, which is the Pro12Ala coding variant and was found to be associated with decreased T2D risk. ${ }^{33}$ We found the association of this SNP was in the same direction as was found by Altshuler et $a l{ }^{33}$ the minor allele was protective against T2D when defined by $\mathrm{HbAlc} \geq 6.5 \%$. We did not find association of the variants in the two previously implicated T2D loci, CDKAL1 and SLC30A8, with FPG, HbA1c or insulin levels. Our study failed to confirm the association between variants in $M C 4 R$ and measures of body fatness. The MC4R SNPs were found to be associated with fat mass, Wt, WC, insulin resistance in previous GWAS. ${ }^{12,13}$ We also did not find association of the INSIG2 SNP, rs7566605, with any of the phenotypic traits. This was the first variant implicated in obesity in a GWAS. ${ }^{8}$
However, its association remains largely inconclusive. ${ }^{34}$ Failure to replicate these findings, whose effect sizes are small, could have stemmed from insufficient sample size. It should also be noted that the GWAS SNPs may not be the true trait or disease-specific variants, rather these could be in LD with the causal variants. Varied patterns of LD across populations owing to genetic diversity could mask the association of an index SNP with the trait in different populations. We report nominal associations of the GWAS variants with several other metabolic traits, which have not yet been explored in other studies. Therefore, these results need further verification in larger samples of varied ancestries.

\section{CONFLICT OF INTEREST}

The authors declare no conflict of interest.

\section{ACKNOWLEDGEMENTS}

This study was supported by a grant from the National Institutes of Health, USA (R01 DK069845) and grants from the Croatian Ministry of Science, Education and Sports (196-1962766-2751 to PR, 196-1962766-2747 to NSN, 196-0342282-0291 to ZD). RK was supported by a training grant from the National Institute of Environmental Health Sciences, USA (T32 ES010957). GZ was supported partially by a gift fund from Ellen and the late Dr George Rieveschl Jr.

\section{AUTHOR CONTRIBUTIONS}

The study was conceived and designed by RD, PR and RC. Sample and data collection was conducted by NJ, DH-A, SM, NSN, ZD and PR. Genotyping was performed by RK, SRI and GS. Statistical analysis was performed by RK, GZ, WN and RC. The draft paper was prepared by RK, GZ, ZD, NSN, RC, PR and RD. All authors read and approved the final draft.

1 Sladek R, Rocheleau G, Rung J et al: A genome-wide association study identifies novel risk loci for type 2 diabetes. Nature 2007; 445: 881-885.

2 Scott LJ, Mohlke KL, Bonnycastle LL et al: A genome-wide association study of type 2 diabetes in Finns detects multiple susceptibility variants. Science 2007; 316: 1341-1345.

3 Diabetes Genetics Initiative of Broad Institute of Harvard and MIT, Lund University, and Novartis Institutes of BioMedical Research: Genome-wide association analysis identifies loci for type 2 diabetes and triglyceride levels. Science 2007; 316: 1331-1336.

4 Zeggini E, Weedon MN, Lindgren CM et al: Replication of genome-wide association signals in UK samples reveals risk loci for type 2 diabetes. Science 2007; 316: 1336-1341.

5 Steinthorsdottir V, Thorleifsson G, Reynisdottir I et al: A variant in CDKAL1 influences insulin response and risk of type 2 diabetes. Nat Genet 2007; 39: 770-775.

6 Gudmundsson J, Sulem P, Steinthorsdottir V et al: Two variants on chromosome 17 confer prostate cancer risk, and the one in TCF2 protects against type-2 diabetes. Nat Genet 2007; 39: 977-983.

7 The Wellcome Trust Case Control Consortium: Genome-wide association study of 14000 cases of seven common diseases and 3000 shared controls. Nature 2007; 447: 661-678

8 Herbert A, Gerry NP, McQueen MB et al: A common genetic variant is associated with adult and childhood obesity. Science 2006; 312: 279-283.

9 Frayling TM, Timpson NJ, Weedon MN et al: A common variant in the FTO gene is associated with body mass index and predisposes to childhood and adult obesity. Science 2007; 316: 889-894.

10 Dina C, Meyre D, Gallina S et al: Variation in FTO contributes to childhood obesity and severe adult obesity. Nat Genet 2007; 39: 724-726.

11 Hinney A, Nguyen TT, Scherag A et al: Genome wide association (GWA) study for early onset extreme obesity supports the role of fat mass and obesity associated gene (FTO) variants. PLoS One 2007; 2: e1361.

12 Loos RJ, Lindgren CM, Li S et al: Common variants near MC4R are associated with fat mass, weight and risk of obesity. Nat Genet 2008; 40: 768-775.

13 Chambers JC, Elliott $\mathrm{P}$, Zabaneh $\mathrm{D}$ et al: Common genetic variation near MC4R is associated with waist circumference and insulin resistance. Nat Genet 2008; 40: 716-718.

14 Thorleifsson G, Walters GB, Gudbjartsson DF et al: Genome-wide association yields new sequence variants at seven loci that associate with measures of obesity. Nat Genet 2009; 41: 18-24. 
15 Frayling TM: Genome-wide association studies provide new insights into type 2 diabetes aetiology. Nat Rev Genet 2007; 8: 657-662.

16 Zeggini E, Scott LJ, Saxena R et al: Meta-analysis of genome-wide association data and large-scale replication idenitifies additional susceptibility loci for type 2 diabetes. Nat Genet 2008; 40: 638-645.

17 Rudan P, Roberts DF, Sujoldzic A et al: Strategy of anthropological research on the island of Hvar. Coll Anthropologicum 1982; 6: 39-46.

18 Rudan P, Sujoldzic, Simic D et al: Population structure in eastern Adriatic: the influence of historical processes, migration patterns, isolation and ecological pressures, and their interaction. in Roberts DF, Fujiki N, Torizuka K (eds): Isolation, Migration and Health. Cambridge University Press: Cambridge (SSHB), 1992, pp 204-218.

19 Smolej-Narancic N, Zagar I: Overweight and fatness in Dalmatia, Croatia: comparison with the US population reference. Coll Antropol 2000; 24: 411-421.

20 Rudan I, Rudan D, Campbell $\mathrm{H}$ et al: Inbreeding and risk of late onset complex disease. J Med Genet 2003; 40: 925-932.

21 Pucarin-Cvetkovic J, Mustajbegovic J, Jelinic JD et al: Body mass index and nutrition as determinants of health and disease in population of Croatian Adriatic islands. Croat Med J 2006; 47: 619-626.

22 Pal P, Xi H, Sun G et al: Tagging SNPs in the kallikrein genes 3 and 2 on 19q13 and their associations with prostate cancer in men of European origin. Hum Genet 2007; 122: 251-259.

23 National Institutes of Health: Third Report of the National Cholesterol Education Program Expert Panel on Detection, Evaluation, and Treatment of High Blood Cholesterol in Adults (Adult Treatment Panel III). National Institutes of Health, NIH Publication No 01-3670, Bethesda, MD, 2001.

24 International Diabetes Federation: The IDF consensus worldwide definition of the metabolic syndrome. April 2005, http://www.idf.org/webdata/docs/MetSyndrome_ Final.pdf.
25 Scuteri A, Sanna S, Chen WM et al: Genome-wide association scan shows genetic variants in the FTO gene are associated with obesity-related traits. PLoS Genet 2007; 3: e115.

26 Grant SF, Li M, Bradfield JP et al: Association analysis of the FTO gene with obesity in children of Caucasian and African ancestry reveals a common tagging SNP. PLoS One 2008; 3: e1746.

27 Price RA, Li W-D, Zhao H: FTO gene SNPs associated with extreme obesity in cases, controls and extremely discordant sister pairs. BMC Med Genet 2008; 9: 4.

28 Willer CJ, Speliotes EK, Loos RJ et al: Six new loci associated with body mass index highlight a neuronal influence on body weight regulation. Nat Genet 2009; 41: 25-34.

29 Zhang G, Karns R, Narancic NS et al: Common SNPs in FTO gene are associated with obesity related anthropometric traits in an island population from the eastern Adriatic coast of Croatia. PLoS One 2010; 5: e10375.

30 Grant SF, Thorleifsson G, Reynisdottir I et al: Variant of transcription factor 7-like 2 (TCF7L2) gene confers risk of type 2 diabetes. Nat Genet 2006; 38: 320-323.

31 Cauchi S, El Achhab Y, Choquet $\mathrm{H}$ et al: TCF7L2 is reproducibly associated with type 2 diabetes in various ethnic groups: a global meta-analysis. J Mol Med 2007; 85: 777-782.

32 Tong $\mathrm{Y}$, Lin $\mathrm{Y}$, Zhang $\mathrm{Y}$ et al: Association between TCF7L2 gene polymorphisms and susceptibility to type 2 diabetes mellitus: a large human genome epidemiology (HuGE) review and meta-analysis. BMC Med Genet 2009; 10: 15.

33 Altshuler D, Hirschhorn JN, Klannemark M et al: The common PPARgamma Pro12Ala polymorphism is associated with decreased risk of type 2 diabetes. Nat Genet 2000; 26: $76-80$.

34 Lyon HN, Emilsson V, Hinney A et al: The association of a SNP upstream of INSIG2 with body mass index is reproduced in several but not all cohorts. PLoS Genet 2007; 3: e61.

Supplementary Information accompanies the paper on European Journal of Human Genetics website (http://www.nature.com/ejhg) 